\title{
Water storage and early hydrous melting of the Martian mantle
}

\author{
A. Pommier*, T.L. Grove, B. Charlier \\ Department of Earth, Atmospheric and Planetary Sciences, Massachusetts Institute of Technology, Cambridge, MA 02139, USA
}

\section{A R T I C L E I N F O}

\section{Article history:}

Received 5 August 2011

Received in revised form

17 January 2012

Accepted 25 March 2012

Editor: T. Spohn

\section{Keywords:}

Mars

early accretion

hydrous phases

phase equilibria

multi-anvil experiments

\begin{abstract}
A B S T R A C T
We report an experimental investigation of the near-solidus phase equilibria of a water-saturated analog of the Martian mantle. Experiments were performed at low temperatures $\left(700-920^{\circ} \mathrm{C}\right)$ and high pressure (4-7 GPa) using multi-anvil apparatus and piston cylinder device (4 GPa). The results of this study are used to explore the role of water during early melting and chemical differentiation of Mars, and to further our understanding of the near-solidus behavior in planetary mantle compositions at high pressure. Water has a significant effect on the temperature of melting and, therefore, on accretion and subsequent differentiation processes. Experiments locate the wet solidus at $\sim 800^{\circ} \mathrm{C}$, and is isothermal between $4 \mathrm{GPa}$ and $7 \mathrm{GPa}$. The Martian primitive mantle can store significant amounts of water in hydrous minerals stable near the solidus. Humite-group minerals and phase E represent the most abundant hydrous minerals stable in the 4-7 GPa pressure range. The amount of water that can be stored in the mantle and mobilized during melting ranges from 1 to up to $4 \mathrm{wt} \%$ near the wet solidus. We discuss thermal models of Mars accretion where the planet formed very rapidly and early on in solar system history. We incorporate the time constraint of Dauphas and Pourmand (2011) that Mars had accreted to $50 \%$ of its present mass in $1.8 \mathrm{Myr}$ and include the effects of ${ }^{26} \mathrm{Al}$ radioactive decay and heat supplied by rapid accretion. When accretion has reached $30 \%$ of Mars current mass ( $\sim 70 \%$ of its present size), melting starts, and extends from 100 to $720 \mathrm{~km}$ depth. Below this melt layer, water can still be bound in crystalline solids. The critical stage is at $50 \%$ accretion ( $~ 80 \%$ of its size), where Mars is above the wet and dry solidi with most of its interior melted. This is earlier in the accretion process than what would be predicted from dry melting. We suggest that water may have promoted early core formation on Mars and rapidly extended melting over a large portion of Mars interior.
\end{abstract}

(c) 2012 Elsevier B.V. All rights reserved.

\section{Introduction}

Geological evidence points to the presence of water on the surface of Mars throughout the history of the planet (Carr and Head, 2010). However, there is no consensus on when and how water has been added to the planet and on its role in the early chemical differentiation of Mars (e.g. Lunine et al., 2003; Drake, 2005). If water was added at the very beginning of accretion from planetesimals, then it was likely stored in mineral phases. To remain in Mars as the planet grows, water must survive heating from energy released during impacts and radioactive heating. Thermal models of Mars allow sufficiently low temperatures at the beginning of accretion for water to be bound into mineral phases (e.g. Senshu et al., 2002) and melting powered by the heat generated from ${ }^{26} \mathrm{Al}$ decay (Dauphas and Pourmand, 2011) will ultimately redistribute and drive volatiles out of the proto-mantle (Marty and Marti, 2002).

\footnotetext{
* Corresponding author. Now at: School of Earth and Space Exploration, Arizona State University, Tempe, AZ 85287, USA. Tel.: +1 4809654214.

E-mail address: Anne.Pommier@asu.edu (A. Pommier).
}

Quantifying the amount of water initially stored in the planetary embryo places important constraints on the next steps of its accretion, mainly the formation and evolution of the magma ocean, the differentiation into a core and the degassing of the planet's interior that led to the formation of a proto-atmosphere. The total abundance of water throughout the history of Mars remains an outstanding question of Martian geology (e.g. Carr and Head, 2010). Water estimates for the melts derived from the Martian mantle range from 1 ppm (Mysen et al., 1998) to $1.8 \mathrm{wt} \%$ (McSween et al., 2001) and estimates of the water added by accreting bodies indicate that as much as $25 \%$ of one Earth ocean $\left(0.38 \times 10^{20} \mathrm{~kg}\right.$, Lunine et al., 2003) could have been added. Experiments in the laboratory on Martian meteorites (shergottite-nakhlite-chassignite (SNC) meteorites) and mantle analog compositions represent a reliable source of information about igneous processes and phase-relationships. These data are the filter through which computing models attempt to constrain accretion and differentiation processes of Mars.

The post-magma ocean history of Mars has been widely investigated with experiments on a dry Martian mantle and basalt analogs (Bertka and Holloway, 1994a,b; Bertka and Fei, 1997; Agee and Draper, 2004; Musselwhite et al., 2006; Monders 
et al., 2007; Minitti and Rutherford, 2007; Filiberto et al., 2008, 2010; Filiberto and Treiman, 2009). However, only a limited number of studies performed on a hydrous mantle analog at experimental conditions relevant to early water storage on Mars investigated the pre-magma ocean time. Water-saturated experiments by Médard and Grove (2006) at lower temperature (775$1000{ }^{\circ} \mathrm{C}$ ) and over the pressure range $1-3 \mathrm{GPa}$ quantified the significant amount of water (up to more than $1 \mathrm{wt} \%$ ) that can be stored in a mantle made of hydrous minerals (chlorite, amphibole and antigorite). The observed effect of water on the solidus of the mantle also allowed them to deduce that hydrous melting probably started when the planet accreted to $\sim 70 \%$ of its present size. $70 \%$ of Mars size corresponds to a radius of $\sim 2365 \mathrm{~km}$, which justifies the need to investigate the properties of a hydrous mantle at higher pressures than $3 \mathrm{GPa}$.

This paper presents an experimental investigation at low temperature $\left(700-920^{\circ} \mathrm{C}\right.$ ) and high pressure (4-7 GPa) of a water-saturated analog of the primitive Martian mantle (Dreibus and Wänke, 1985). This composition corresponds to the mantle+crust Martian bulk composition and is consistent with phase relations of basaltic rocks from Gusev crater (Monders et al., 2007). The hypothesis of an accretion-driven early hydrous melting is tested in order to further our understanding of the processes by which Mars evolved to its present state.

\section{Experimental and analytical methods}

\subsection{Starting bulk composition}

Experiments have been performed on the Martian mantle analog determined by Dreibus and Wänke (1985) (Table 1). This bulk composition was estimated using the elemental abundances in SNC meteorites and evidence from the $\mathrm{Mg}$, Si and refractory lithophile element abundances in $\mathrm{CI}$ chondrites to infer a composition for the other major elements and volatile trace elements. This composition is similar to the one proposed by Lodders and Fegley (1997) from oxygen isotope evidence. Also, the Dreibus and Wänke (1985) composition has been shown to be consistent with phase equilibria constraints from basaltic rocks at Gusev crater (Bertka and Holloway, 1994; Monders et al., 2007), and it allows a direct comparison with the work by Médard and Grove (2006) performed at lower pressures. Bertka and Fei (1997) have also investigated the phase equilibrium of this composition over a wide pressure range under dry conditions (e.g. Bertka and Fei,

Table 1

Starting composition (Dreibus and Wänke (1985), DW) (wt\%) and comparison with terrestrial upper mantle (Hart and Zindler, 1986).

\begin{tabular}{lcc}
\hline & Mars & Earth \\
\hline $\mathrm{SiO}_{2}$ & 44.5 & 46.1 \\
$\mathrm{TiO}_{2}$ & 0.10 & 0.18 \\
$\mathrm{Al}_{2} \mathrm{O}_{3}$ & 3.01 & 4.07 \\
$\mathrm{Cr}_{2} \mathrm{O}_{3}$ & 0.80 & 0.47 \\
$\mathrm{FeOt}$ & 17.9 & 7.56 \\
$\mathrm{MnO}$ & 0.50 & 0.13 \\
$\mathrm{MgO}$ & 30.3 & 37.9 \\
$\mathrm{CaO}$ & 2.41 & 3.22 \\
$\mathrm{Na}_{2} \mathrm{O}$ & 0.50 & 0.33 \\
$\mathrm{~K}_{2} \mathrm{O}$ & 0.04 & 0.03 \\
$\mathrm{P}_{2} \mathrm{O}_{5}$ & 0.16 & 0.02 \\
$\mathrm{Mg}^{\# \mathrm{a}}$ & 75 & 90 \\
\hline
\end{tabular}

${ }^{\mathrm{a}}$ molar $\mathrm{Mg} /(\mathrm{Mg}+\mathrm{Fe})$.
1997), allowing us to compare anhydrous and water-bearing phase equilibria.

The starting material consists of a water-saturated Dreibus and Wänke (1985) composition (abbreviated DWH), obtained from analytical grade oxides mixing. The amount of water added under the form of brucite $\left(\mathrm{Mg}(\mathrm{OH})_{2}\right)$ is high enough to ensure $\mathrm{H}_{2} \mathrm{O}$-saturation over the experimental pressure conditions. Details regarding the starting material preparation are presented in Médard and Grove (2006).

\subsection{Experimental conditions and calibrations}

Water-saturated experiments have been conducted between 4 and $7 \mathrm{GPa}$, and at temperatures between 700 and $920^{\circ} \mathrm{C}$. Run durations range from 2.5 up to 11 days and the lower-temperature experiments had the longest durations (Table 2). Experiments at 4 GPa have been performed with a $12.7 \mathrm{~mm}$ end-loaded solidmedium piston-cylinder apparatus (Boyd and England, 1960) using the hot piston-in technique. Experimental details for the piston-cylinder experiments are discussed in Médard and Grove (2006). Experiments at higher pressures have been conducted in a multi-anvil apparatus. The design of our multi-anvil device is similar to that of Walker (1991) and a 12/8 (octahedron edge length/truncated edge length, in $\mathrm{mm}$ ) cell assembly was used (Supplementary materials).

Pressures were calibrated at $1000{ }^{\circ} \mathrm{C}$ using the quartz-coesite transition at 3.1 GPa (Bohlen and Boettcher, 1982) and at $1200{ }^{\circ} \mathrm{C}$ using the fayalite-spinel transition at $5.75 \mathrm{GPa}$ (Yagi et al., 1987). In both cases, minerals were identified according to their respective characteristic refractive indices. We performed a two-pyroxene thermometry experiment to determine the temperature gradient in our sectioned graphite furnace assembly (Lindsley and Dixon, 1976) as well as an experiment with two thermocouples, placed on both sides of the capsule. In both cases, temperature gradient across the capsule is estimated to be a few tens of Celsius degrees, in agreement with predictions using the thermal modeling program by Hernlund et al. (2006) (Supplementary materials S1).

For each experiment, the approach to equilibrium is enhanced by the presence of a free fluid phase. We consider that chemical equilibrium was attained because crystals do not present any chemical zoning. Also, two experiments (one in piston-cylinder and one in multi-anvil apparatus) were carried out under identical conditions to demonstrate the compatibility of the results from the two experimental devices (Table 2).

\subsection{Mineral identification and analytical technique}

Longitudinal sections of the experimental charges were mounted and polished. Backscattered electron images, chemical composition maps and quantitative chemical analyses were obtained with a five spectrometer JEOL 8200 electron microprobe using wavelength dispersive spectrometry (WDS) at the electron microprobe facility at MIT. Analyses were performed with a $15 \mathrm{kV}$ accelerating voltage and a beam current of $10 \mathrm{nA}$. All mineral phases were analyzed with a focused beam and quenched melt analyses were analyzed with a $10 \mu \mathrm{m}$ beam. Natural and synthetic primary and secondary standards were used and the CITZAF online data correction package was used for all analyses (Armstrong, 1995).

Because many phases have grain size $<5 \mu \mathrm{m}$ (even for the longest run durations), quantitative analysis was challenging because analyses sometimes were a composite of two different adjacent grains. We solved this problem performing multiple analyses of all candidate minerals until clear subsets of microprobe analyses emerged and defined distinct, stoichiometrically 
Table 2

Summary of experimental conditions and phase proportions.

\begin{tabular}{|c|c|c|c|c|}
\hline Run & $\begin{array}{l}T \\
\text { (deg.) }\end{array}$ & $\begin{array}{l}P \\
(\text { GPa })\end{array}$ & $\begin{array}{l}\text { Duration } \\
\text { (days) }\end{array}$ & $\begin{array}{l}\text { Phases } \\
\text { (vol\%) }\end{array}$ \\
\hline D242 & 760 & 4 & 8 & ol(30), opx(12), $\operatorname{cpx}(27), \operatorname{gt}(10), \operatorname{ap}(\operatorname{tr}), \operatorname{sp}(1),(\mathrm{Ti}) \operatorname{chum}(19), \operatorname{chl}(1)$ \\
\hline D235 & 800 & 4 & 8 & ol(41), opx(35), cpx(5), gt(14), ap(tr), sp(tr), (Ti)chum(3), gl(2) \\
\hline $\mathrm{D} 240^{\mathrm{a}}$ & 800 & 4 & 8 & ol(41), opx(35), cpx(5), gt(14), ap(tr), sp(tr), (Ti)chum(3), gl(2) \\
\hline A40 & 780 & 5 & 10 & ol(12), opx(49), $\operatorname{cpx}(1), \operatorname{gt}(13), \operatorname{Tichum}(10), \operatorname{phE}(15)$ \\
\hline A26 & 720 & 5.2 & 5 & $\operatorname{ol}(5), \operatorname{opx}(28), \operatorname{cpx}(7), \operatorname{gt}(12), \operatorname{sp}(2),(\mathrm{Ti}) \operatorname{chum}(36)$ \\
\hline A25 & 920 & 5.2 & 2.5 & ol(53) opx(19), cpx(8), gt(6), sp(5), gl(9) \\
\hline A34 & 850 & 6 & 6 & $\mathrm{ol}(47), \mathrm{opx}(31), \mathrm{cpx}^{\mathrm{b}}(\operatorname{tr}), \mathrm{gt}(15), \mathrm{sp}(\operatorname{tr}), \operatorname{chum}(6), \mathrm{gl}(1)$ \\
\hline A31 & 720 & 6.5 & 11 & $\mathrm{ol}(31), \operatorname{opx}(31), \operatorname{cpx}(11), \mathrm{gt}(14), \mathrm{ap}(\mathrm{tr}), \mathrm{sp}(\operatorname{tr}), \operatorname{chum}(\operatorname{tr}), \operatorname{phE}(13)$ \\
\hline A27 & 820 & 6.5 & 4 & $\mathrm{ol}^{\mathrm{b}}(2), \operatorname{opx}(40), \operatorname{cpx}(5), \mathrm{gt}(15), \mathrm{sp}(2), \operatorname{phE}(36)$ \\
\hline $\mathrm{A} 39^{\mathrm{a}}$ & 820 & 6.5 & 8 & $\mathrm{ol}^{\mathrm{b}}(2), \operatorname{opx}(40), \operatorname{cpx}(5), \operatorname{gt}(15), \operatorname{sp}(2), \operatorname{phE}(36)$ \\
\hline A36 & 770 & 7 & 8.5 & ol(38), opx(22), cpx(5), gt(15), sp(tr), phE(20) \\
\hline A38 & 850 & 7 & 4 & $\mathrm{ol}^{\mathrm{b}}(2), \operatorname{opx}(27), \operatorname{cpx}(18), \mathrm{gt}(15), \mathrm{sp}(\operatorname{tr}), \mathrm{phE}(38)$ \\
\hline
\end{tabular}

a Reproducibility experiments.

${ }^{\mathrm{b}}$ No microprobe analyses, phase identified using EDS spectra.

coherent mineral compositions. This technique, together with Raman analyses on selected samples was used to verify the identity of mineral groups in each experimental sample.

Water contents in hydrous phases were estimated using the summation deficit from the electron microprobe analysis (difference between original analytical total and a sum of $100 \mathrm{wt} \%$ gives $\mathrm{H}_{2} \mathrm{O}$ content of the mineral). This technique was checked for selected hydrous phases, by combining identification from electron microprobe WDS analyses and qualitative EDS identification with additional analyses using Raman spectroscopy performed at the University of Minnesota. Existing spectra of hydrous minerals (e.g. Mernagh and Liu, 1998; Frost et al., 2007) were used as fingerprints to confirm the identity of the phases in the experimental samples (Supplementary material S2).

\section{Results}

The assemblages produced in our experiments are listed in Table 2. Mineral and melt analyses are presented in Table 3.

\subsection{Nominally anhydrous phases}

Olivine, orthopyroxene, clinopyroxene and garnet are present in all experiments. The composition of olivine ranges from Fo75 to Fo80; the Mg\# of orthopyroxene is $0.78-0.88$. Spinel occurs in all experiments but the small size of the euhedral crystals allowed measurements in 4 samples only. The compositions lie close to the chromite-magnetite solid solution. The spinel also contains minor amounts of $\mathrm{Al}_{2} \mathrm{O}_{3}$ (0.5-6.9 wt\%), $\mathrm{MgO}$ (2.1-3.5 wt\%) and $\mathrm{TiO}_{2}$ (0.3-3.2 wt\%). Two experiments contain two distinct populations that lie across the miscibility gap between in the chromite-magnetite binary system (Sack and Ghiorso, 1991).

\subsection{Wet solidus and hydrous melt}

Over the investigated pressure range, the wet solidus has been crossed at $\sim 800{ }^{\circ} \mathrm{C}$. Once the average mantle geotherm of the accreting planet passes this reaction, a hydrous melt is formed under water-saturated conditions. Our phase diagram in Fig. 1 shows that wet solidus reaction is not affected by pressure above $\sim 3 \mathrm{GPa}$. The experiment is considered to contain melt when glass or quench crystals are observed in backscattered electron images. The quench glass is difficult to analyze because of the presence of quench crystals. Moreover, in most partially melted runs, glass is present in very small amount and concentrated on grain boundaries, as previously observed by Médard and Grove (2006). However, in one run (A25), a few large glass pockets segregated from the crystals, allowing microprobe analyses (Fig. 2). The composition is silica-rich, with $\sim 77 \mathrm{wt} \% \mathrm{SiO}_{2}$ and $3.6 \mathrm{wt} \% \mathrm{Na}_{2} \mathrm{O}+\mathrm{K}_{2} \mathrm{O}$ (on an anhydrous basis, Table 3 ), which is consistent with the fact that low degrees of partial melting of peridotite can produce high silica melts (e.g. Kushiro et al., 1968).

\subsection{Hydrated minerals stable under pressure}

Depending on the experimental conditions, different hydrous minerals have been identified, their water content ranging from 4 to $\sim 13$ wt\%.

Chlorite has been identified using EDS spectra in only one run $\left(760{ }^{\circ} \mathrm{C}, 4 \mathrm{GPa}\right)$, which places an important constraint on its stability field (Fig. 1). This phase was present in very small amount in the sample, rendering its chemical analyses difficult. However, its composition was found to be similar to the one analyzed at a similar temperature and $3 \mathrm{GPa}$ by Médard and Grove (2006).

Humite minerals have been identified in all our runs below $850{ }^{\circ} \mathrm{C}$ and $6 \mathrm{GPa}$ (Fig. 1 and Table 2). The presence of minerals that are part of the humite family was expected since they have stability fields below $13 \mathrm{GPa}$ and below $1000{ }^{\circ} \mathrm{C}$ (e.g. Kawamoto et al., 1996). These minerals are structurally similar to olivine with interlayers of brucite $\left(\mathrm{Mg}(\mathrm{OH})_{2}\right)$ their formula being $\mathrm{n}(\mathrm{Mg}, \mathrm{Fe})_{2} \mathrm{SiO}_{4}$. $(\mathrm{Mg}, \mathrm{Fe})(\mathrm{OH}, \mathrm{F})_{2}$, in which $\mathrm{n}$ values of 2 and 4 correspond to chondrodite and clinohumite, respectively. At pressures up to $5.2 \mathrm{GPa}$, the presence of titanoclinohumite was also observed (Table 2).

Phase $\mathrm{E}$ has been identified in the highest pressure experiments $(P>4.5 \mathrm{GPa})$ and represents with humites the other most abundant hydrous mineral in this study (Fig. 1 and Table 2). Phase $\mathrm{E}$ $\left(\mathrm{Mg}_{2.3} \mathrm{Si}_{1.25} \mathrm{H}_{2.4} \mathrm{O}_{6}\right)$ is a high-pressure and dense hydrous magnesium silicate (DHMS) whose water storage capacity can be as high as 15.8 wt\% $\mathrm{H}_{2} \mathrm{O}$ (e.g. Kudoh et al., 1993). Chemical variations in the composition of phase $\mathrm{E}$ have been observed in the same experiment (ternary diagrams, Fig. 1), which is consistent with other previous experimental investigations (Kudoh et al., 1993). In Earth mantleanalog compositions, phase $\mathrm{E}$ is stable at higher pressure (12$15 \mathrm{GPa}$ ) and higher temperatures $\left(800-1100{ }^{\circ} \mathrm{C}\right.$ ) (Kanzaki, 1989, 1991). Neither phase $\mathrm{A}\left(\mathrm{Mg}_{7} \mathrm{Si}_{2} \mathrm{O}_{8}(\mathrm{OH})_{6}, 12 \mathrm{wt} \% \mathrm{H}_{2} \mathrm{O}\right.$, Horiuchi et al., 1979) nor the $10 \AA$ phase $\left(\mathrm{Mg}_{3} \mathrm{Si}_{4} \mathrm{O}_{14} \mathrm{H}_{6}, 13 \mathrm{wt} \% \mathrm{H}_{2} \mathrm{O}\right.$, Comodi et al., 2007) have been observed in our Martian mantle analog experiments, while these two DHMS have stabilities corresponding to the 
Table 3

Electron microprobe analyses of run products.

\begin{tabular}{|c|c|c|c|c|c|c|c|c|c|c|c|c|c|c|c|c|c|c|c|c|c|c|c|c|c|}
\hline Expt. & $\mathrm{P}(\mathrm{GPa})$ & $\mathrm{T}\left(\left(^{\circ} \mathrm{C}\right)\right.$ & Phase & $\mathrm{SiO}_{2}$ & & $\mathrm{TiO}_{2}$ & & $\mathrm{Al}_{2} \mathrm{O}_{3}$ & & $\mathrm{Cr}_{2} \mathrm{O}_{3}$ & & $\mathrm{FeO}_{\text {tot }}$ & & $\mathrm{MnO}$ & & $\mathrm{MgO}$ & & $\mathrm{CaO}$ & & $\mathrm{Na}_{2} \mathrm{O}$ & & $\mathrm{K}_{2} \mathrm{O}$ & & Mg\# & Sum \\
\hline D242 & 4 & 760 & $\begin{array}{l}\text { ol( }(4)^{\mathrm{a}} \\
\text { opx(12) } \\
\text { cpx(2) } \\
\text { gt(9) } \\
\operatorname{sp}(3) \\
\text { cHum( }(8) \\
\text { TicHum(1) } \\
\text { chl(1) }\end{array}$ & $\begin{array}{l}37.9 \\
54.0 \\
56.4 \\
41.3 \\
0.55 \\
39.7 \\
34.4 \\
27.4\end{array}$ & $\begin{array}{l}(14)^{b} \\
(22) \\
(7) \\
(13) \\
(23) \\
(23)\end{array}$ & $\begin{array}{l}0.06 \\
0.06 \\
0.04 \\
0.19 \\
2.74 \\
0.03 \\
3.04 \\
0.01\end{array}$ & $\begin{array}{l}(6) \\
(2) \\
(1) \\
(8) \\
(3) \\
(2)\end{array}$ & $\begin{array}{l}0.07 \\
2.19 \\
2.26 \\
19.6 \\
6.94 \\
0.59 \\
0.14 \\
13.8\end{array}$ & $\begin{array}{l}(8) \\
(34) \\
(33) \\
(12) \\
(27) \\
(33)\end{array}$ & $\begin{array}{l}- \\
0.18 \\
0.33 \\
0.01 \\
38.2 \\
- \\
- \\
0.00\end{array}$ & $\begin{array}{l}(5) \\
(3) \\
(2) \\
(32)\end{array}$ & $\begin{array}{l}21.5 \\
13.7 \\
11.9 \\
19.5 \\
42.1 \\
20.5 \\
17.9 \\
13.3\end{array}$ & $\begin{array}{l}(13) \\
(12) \\
(1) \\
(7) \\
(7) \\
(10)\end{array}$ & $\begin{array}{l}0.28 \\
0.36 \\
0.31 \\
0.63 \\
0.43 \\
0.28 \\
0.19 \\
0.12\end{array}$ & $\begin{array}{l}(9) \\
(5) \\
(3) \\
(13) \\
(6) \\
(7)\end{array}$ & $\begin{array}{l}41.4 \\
28.9 \\
23.1 \\
10.5 \\
3.50 \\
35.1 \\
43.7 \\
24.7\end{array}$ & $\begin{array}{l}(12) \\
(15) \\
(15) \\
(13) \\
(64) \\
(17)\end{array}$ & $\begin{array}{l}0.04 \\
0.57 \\
5.51 \\
8.50 \\
0.06 \\
0.14 \\
0.17 \\
0.24\end{array}$ & $\begin{array}{l}(3) \\
(11) \\
(42) \\
(8) \\
(1) \\
(10)\end{array}$ & $\begin{array}{l}\overline{-} \\
0.10 \\
0.11 \\
0.01 \\
- \\
- \\
\overline{0} \\
0.11\end{array}$ & $\begin{array}{l}\text { (3) } \\
(3) \\
(2)\end{array}$ & $\begin{array}{l}- \\
0.01 \\
0.01 \\
- \\
- \\
- \\
- \\
-\end{array}$ & $\begin{array}{l}(1) \\
(0)\end{array}$ & $\begin{array}{l}0.77 \\
0.79 \\
0.78 \\
0.49\end{array}$ & $\begin{array}{l}101.3 \\
100.0 \\
100.0 \\
100.3 \\
94.6 \\
96.4 \\
99.6 \\
79.7\end{array}$ \\
\hline $\begin{array}{l}\text { D235 } \\
\text { D240 }\end{array}$ & 4 & 800 & $\begin{array}{l}\text { ol(19) } \\
\text { opx(10) } \\
\operatorname{cpx}(8) \\
\operatorname{gt}(4) \\
\operatorname{cHum}(3)\end{array}$ & $\begin{array}{l}38.4 \\
53.8 \\
53.7 \\
40.3 \\
41.7\end{array}$ & $\begin{array}{l}(11) \\
(22) \\
(15) \\
(7) \\
(22)\end{array}$ & $\begin{array}{l}0.12 \\
0.05 \\
0.03 \\
0.41 \\
0.04\end{array}$ & $\begin{array}{l}(25) \\
(4) \\
(2) \\
(6) \\
(2)\end{array}$ & $\begin{array}{l}0.16 \\
1.54 \\
0.75 \\
17.0 \\
0.60\end{array}$ & $\begin{array}{l}(19) \\
(46) \\
(53) \\
(7) \\
(11)\end{array}$ & $\begin{array}{l}- \\
0.21 \\
0.40 \\
0.01 \\
-\end{array}$ & $\begin{array}{l}(6) \\
(7) \\
(1)\end{array}$ & $\begin{array}{l}22.7 \\
14.0 \\
6.77 \\
18.6 \\
21.3\end{array}$ & $\begin{array}{l}(7) \\
(17) \\
(96) \\
(5) \\
(4)\end{array}$ & $\begin{array}{l}0.33 \\
0.32 \\
0.24 \\
0.59 \\
0.35\end{array}$ & $\begin{array}{l}(4) \\
(3) \\
(3) \\
(2) \\
(5)\end{array}$ & $\begin{array}{l}38.4 \\
28.3 \\
18.2 \\
13.5 \\
31.3\end{array}$ & $\begin{array}{l}(11) \\
(15) \\
(20) \\
(8) \\
(23)\end{array}$ & $\begin{array}{l}0.10 \\
0.91 \\
19.3 \\
8.37 \\
0.17\end{array}$ & $\begin{array}{l}(8) \\
(43) \\
(19) \\
(14) \\
(4)\end{array}$ & $\begin{array}{l}- \\
0.06 \\
0.24 \\
0.02 \\
-\end{array}$ & $\begin{array}{l}\text { (2) } \\
\text { (6) } \\
\text { (1) }\end{array}$ & $\begin{array}{l}\overline{0} \\
0.01 \\
0.01 \\
- \\
-\end{array}$ & $\begin{array}{l}(1) \\
(0)\end{array}$ & $\begin{array}{l}0.75 \\
0.78 \\
0.83 \\
0.56 \\
0.73\end{array}$ & $\begin{array}{l}100.2 \\
99.1 \\
99.6 \\
98.8 \\
95.8\end{array}$ \\
\hline A40 & 5 & 780 & $\begin{array}{l}\text { ol(2) } \\
\text { opx(2) } \\
\operatorname{cpx}(1) \\
\operatorname{gt}(6) \\
\text { TicHum(3) } \\
\text { phE(15) }\end{array}$ & $\begin{array}{l}38.0 \\
53.7 \\
51.0 \\
39.1 \\
33.5 \\
27.4\end{array}$ & $\begin{array}{l}(21) \\
(1) \\
(14) \\
(17) \\
(16)\end{array}$ & $\begin{array}{l}0.26 \\
0.03 \\
0.00 \\
0.46 \\
3.07 \\
0.15\end{array}$ & $\begin{array}{l}(19) \\
(7) \\
(2)\end{array}$ & $\begin{array}{l}0.03 \\
0.57 \\
0.31 \\
17.5 \\
0.03 \\
0.07\end{array}$ & $\begin{array}{l}(5) \\
(2) \\
\\
(14) \\
(2) \\
(3)\end{array}$ & $\begin{array}{l}- \\
0.11 \\
0.39 \\
1.69 \\
- \\
-\end{array}$ & (53) & $\begin{array}{l}20.0 \\
13.6 \\
8.89 \\
16.3 \\
20.9 \\
28.0\end{array}$ & $\begin{array}{l}(14) \\
(3) \\
(6) \\
(4) \\
(4)\end{array}$ & $\begin{array}{l}0.30 \\
0.36 \\
0.54 \\
0.73 \\
0.27 \\
0.38\end{array}$ & $\begin{array}{l}(2) \\
(4) \\
\\
(4) \\
(3) \\
(4)\end{array}$ & $\begin{array}{l}41.9 \\
30.4 \\
21.9 \\
11.5 \\
38.8 \\
32.8\end{array}$ & $\begin{array}{l}(4) \\
(6) \\
(15) \\
(28) \\
(17)\end{array}$ & $\begin{array}{l}0.04 \\
1.24 \\
13.9 \\
11.2 \\
0.03 \\
0.06\end{array}$ & $\begin{array}{l}(13) \\
(1) \\
(9)\end{array}$ & $\begin{array}{l}- \\
0.10 \\
0.43 \\
0.04 \\
- \\
-\end{array}$ & (3) & $\begin{array}{l}- \\
0.01 \\
0.03 \\
- \\
- \\
-\end{array}$ & (0) & $\begin{array}{l}0.79 \\
0.80 \\
0.82 \\
0.56 \\
0.77 \\
0.68\end{array}$ & $\begin{array}{l}100.7 \\
100.1 \\
97.5 \\
98.5 \\
96.6 \\
89.0\end{array}$ \\
\hline A26 & 5.2 & 720 & $\begin{array}{l}\text { ol( }(8) \\
\text { opx(5) } \\
\text { cpx( }(4) \\
\text { gt(9) } \\
\text { sp1(2) } \\
\text { sp2(2) } \\
\text { cHum(2) } \\
\text { TicHum(1) }\end{array}$ & $\begin{array}{l}38.2 \\
57.2 \\
55.4 \\
40.1 \\
0.93 \\
0.92 \\
37.1 \\
34.4\end{array}$ & $\begin{array}{l}(11) \\
(6) \\
(5) \\
(11) \\
(12) \\
(8) \\
(2)\end{array}$ & $\begin{array}{l}0.74 \\
0.01 \\
0.01 \\
0.12 \\
0.27 \\
0.39 \\
0.08 \\
2.87\end{array}$ & $\begin{array}{l}(53) \\
(2) \\
(1) \\
(5) \\
(2) \\
(2) \\
(9)\end{array}$ & $\begin{array}{l}0.31 \\
0.54 \\
0.76 \\
20.2 \\
1.02 \\
0.52 \\
0.29 \\
0.06\end{array}$ & $\begin{array}{l}(51) \\
(18) \\
(8) \\
(15) \\
(38) \\
(55) \\
(33)\end{array}$ & $\begin{array}{l}- \\
0.07 \\
0.20 \\
0.38 \\
31.0 \\
3.49 \\
- \\
-\end{array}$ & $\begin{array}{l}(6) \\
(1) \\
(20) \\
(64) \\
(98)\end{array}$ & $\begin{array}{l}19.6 \\
7.73 \\
66.84 \\
18.4 \\
59.3 \\
84.9 \\
21.4 \\
19.0\end{array}$ & $\begin{array}{l}(10) \\
(9) \\
(16) \\
(7) \\
(49) \\
(13) \\
(4)\end{array}$ & $\begin{array}{l}0.22 \\
0.33 \\
0.75 \\
1.52 \\
0.30 \\
0.14 \\
0.20 \\
0.22\end{array}$ & $\begin{array}{l}(8) \\
(8) \\
(82) \\
(10) \\
(3) \\
(2) \\
(6)\end{array}$ & $\begin{array}{l}41.5 \\
33.2 \\
20.1 \\
12.3 \\
2.12 \\
2.19 \\
38.5 \\
40.8\end{array}$ & $\begin{array}{l}(7) \\
(16) \\
(26) \\
(11) \\
(25) \\
(23) \\
(8)\end{array}$ & $\begin{array}{l}0.20 \\
0.80 \\
15.0 \\
7.69 \\
- \\
- \\
0.06 \\
0.04\end{array}$ & $\begin{array}{l}(29) \\
(34) \\
(23) \\
(8)\end{array}$ & $\begin{array}{l}- \\
0.11 \\
1.18 \\
0.05 \\
0.07 \\
0.44 \\
- \\
-\end{array}$ & $\begin{array}{l}(3) \\
(3) \\
(6) \\
(2) \\
(24)\end{array}$ & $\begin{array}{l}0.01 \\
0.02 \\
- \\
- \\
- \\
- \\
-\end{array}$ & $\begin{array}{l}(1) \\
\text { (1) }\end{array}$ & $\begin{array}{l}0.79 \\
0.88 \\
0.84 \\
0.55 \\
\\
\\
0.76 \\
0.78\end{array}$ & $\begin{array}{l}100.9 \\
99.9 \\
100.3 \\
100.8 \\
95.6 \\
93.2 \\
97.7 \\
97.2\end{array}$ \\
\hline A25 & 5.2 & 920 & $\begin{array}{l}\text { ol(7) } \\
\text { opx(8) } \\
\operatorname{cpx}(6) \\
\operatorname{gt}(12) \\
\text { sp1(3) } \\
\text { sp2(7) } \\
\operatorname{gl}(7)\end{array}$ & $\begin{array}{l}38.5 \\
56.6 \\
54.6 \\
40.4 \\
0.61 \\
0.48 \\
59.6\end{array}$ & $\begin{array}{l}(6) \\
(6) \\
(9) \\
(7) \\
(15) \\
(20) \\
(4)\end{array}$ & $\begin{array}{l}0.05 \\
0.03 \\
0.02 \\
0.18 \\
1.29 \\
1.66 \\
0.04\end{array}$ & $\begin{array}{l}(10) \\
(2) \\
(1) \\
(2) \\
(6) \\
(7) \\
(3)\end{array}$ & $\begin{array}{l}0.04 \\
0.52 \\
0.46 \\
19.7 \\
1.20 \\
0.93 \\
12.6\end{array}$ & $\begin{array}{l}(5) \\
(9) \\
(4) \\
(6) \\
(8) \\
(10) \\
(4)\end{array}$ & $\begin{array}{l}- \\
0.21 \\
0.22 \\
0.00 \\
9.27 \\
0.99 \\
-\end{array}$ & $\begin{array}{l}(6) \\
(6) \\
(1) \\
(21) \\
(67)\end{array}$ & $\begin{array}{l}18.8 \\
10.9 \\
5.56 \\
18.7 \\
77.2 \\
86.4 \\
1.19\end{array}$ & $\begin{array}{l}(9) \\
(5) \\
(30) \\
(6) \\
(4) \\
(7) \\
(44)\end{array}$ & $\begin{array}{l}0.30 \\
0.38 \\
0.29 \\
1.33 \\
0.24 \\
0.16 \\
0.08\end{array}$ & $\begin{array}{l}(5) \\
(3) \\
(4) \\
(5) \\
(1) \\
(4) \\
(4)\end{array}$ & $\begin{array}{l}41.5 \\
31.2 \\
17.1 \\
12.2 \\
2.39 \\
2.24 \\
0.66\end{array}$ & $\begin{array}{l}(8) \\
(8) \\
(5) \\
(6) \\
(6) \\
(28) \\
(21)\end{array}$ & $\begin{array}{l}0.11 \\
0.45 \\
20.4 \\
6.97 \\
- \\
- \\
0.31\end{array}$ & $\begin{array}{l}(19) \\
(17) \\
(14) \\
(6)\end{array}$ & $\begin{array}{l}- \\
0.06 \\
0.53 \\
0.05 \\
0.09 \\
0.20 \\
2.33\end{array}$ & $\begin{array}{l}(3) \\
(4) \\
(4) \\
(8) \\
(15) \\
(46)\end{array}$ & $\begin{array}{l}\overline{-} \\
0.00 \\
0.00 \\
- \\
- \\
\overline{0} \\
0.44\end{array}$ & (1) & $\begin{array}{l}0.80 \\
0.84 \\
0.84 \\
0.54\end{array}$ & $\begin{array}{l}99.3 \\
100.3 \\
99.1 \\
99.9 \\
92.8 \\
93.4 \\
77.3\end{array}$ \\
\hline A34 & 6.0 & 850 & $\begin{array}{l}\text { ol(3) } \\
\text { opx(10) } \\
\text { gt(20) } \\
\text { cHum(5) }\end{array}$ & $\begin{array}{l}39.7 \\
56.8 \\
40.7 \\
38.6\end{array}$ & $\begin{array}{l}(16) \\
(21) \\
(17) \\
(11)\end{array}$ & $\begin{array}{l}0.02 \\
0.06 \\
0.37 \\
0.03\end{array}$ & $\begin{array}{l}(1) \\
(5) \\
(10) \\
(2)\end{array}$ & $\begin{array}{l}0.35 \\
0.43 \\
20.4 \\
0.36\end{array}$ & $\begin{array}{l}(4) \\
(10) \\
(14) \\
(37)\end{array}$ & $\begin{array}{l}0.11 \\
0.52 \\
1.69 \\
0.12\end{array}$ & $\begin{array}{l}(4) \\
(12) \\
(53) \\
(7)\end{array}$ & $\begin{array}{l}22.1 \\
13.0 \\
13.5 \\
22.8\end{array}$ & $\begin{array}{l}(11) \\
(1) \\
(9) \\
(24)\end{array}$ & $\begin{array}{l}0.29 \\
0.35 \\
0.95 \\
0.31\end{array}$ & $\begin{array}{l}(1) \\
(3) \\
(10) \\
(3)\end{array}$ & $\begin{array}{l}37.9 \\
27.7 \\
13.1 \\
31.6\end{array}$ & $\begin{array}{l}(30) \\
(23) \\
(15) \\
(32)\end{array}$ & $\begin{array}{l}0.02 \\
0.40 \\
9.04 \\
0.07\end{array}$ & $\begin{array}{l}(2) \\
(4) \\
(72) \\
(6)\end{array}$ & $\begin{array}{l}0.04 \\
0.02 \\
0.02 \\
0.02\end{array}$ & $\begin{array}{l}(1) \\
(4) \\
(3) \\
(1)\end{array}$ & $\begin{array}{l}0.00 \\
0.00 \\
0.00 \\
0.00\end{array}$ & $\begin{array}{l}(1) \\
(1) \\
(0) \\
(0)\end{array}$ & $\begin{array}{l}0.75 \\
0.79 \\
0.63 \\
0.71\end{array}$ & $\begin{array}{l}100.6 \\
99.2 \\
99.9 \\
94.1\end{array}$ \\
\hline A31 & 6.5 & 720 & $\begin{array}{l}\text { oll(11) } \\
\text { opx(9) } \\
\operatorname{cpx}(1) \\
\text { gt(5) } \\
\text { TicHum(1) } \\
\text { phE(7) }\end{array}$ & $\begin{array}{l}38.2 \\
56.5 \\
52.1 \\
39.7 \\
36.0 \\
28.4\end{array}$ & $\begin{array}{l}(5) \\
(7) \\
(4) \\
(3)\end{array}$ & $\begin{array}{l}0.03 \\
0.01 \\
0.13 \\
0.14 \\
3.00 \\
0.15\end{array}$ & $\begin{array}{l}(3) \\
(1) \\
(6)\end{array}$ & $\begin{array}{l}0.02 \\
0.37 \\
3.20 \\
16.9 \\
0.05 \\
0.58\end{array}$ & $\begin{array}{l}(2) \\
(15) \\
(20) \\
(5)\end{array}$ & $\begin{array}{l}0.12 \\
0.23 \\
0.45 \\
4.19 \\
- \\
0.21\end{array}$ & $\begin{array}{l}(18) \\
(4)\end{array}$ & $\begin{array}{l}20.3 \\
11.7 \\
10.5 \\
18.2 \\
7.73 \\
30.7\end{array}$ & $\begin{array}{l}(7) \\
(17)\end{array}$ & $\begin{array}{l}0.29 \\
0.34 \\
0.38 \\
0.72 \\
0.09 \\
0.62\end{array}$ & $\begin{array}{l}(3) \\
(6) \\
(5) \\
(10)\end{array}$ & $\begin{array}{l}40.4 \\
30.6 \\
17.6 \\
15.5 \\
48.1 \\
28.0\end{array}$ & $\begin{array}{l}(8) \\
(12) \\
(6) \\
(12)\end{array}$ & $\begin{array}{l}0.05 \\
0.64 \\
14.9 \\
3.91 \\
0.04 \\
0.16\end{array}$ & $\begin{array}{l}(2) \\
(6) \\
(5) \\
(7)\end{array}$ & $\begin{array}{l}0.08 \\
0.06 \\
0.47 \\
0.00 \\
- \\
0.08\end{array}$ & $\begin{array}{l}(3) \\
\text { (4) } \\
(0) \\
(2)\end{array}$ & $\begin{array}{l}- \\
- \\
- \\
- \\
- \\
-\end{array}$ & & $\begin{array}{l}0.78 \\
0.82 \\
0.75 \\
0.60 \\
0.92 \\
0.62\end{array}$ & $\begin{array}{l}99.5 \\
100.4 \\
99.8 \\
99.1 \\
95.4 \\
89.0\end{array}$ \\
\hline $\begin{array}{l}\text { A27 } \\
\text { A39 }\end{array}$ & 6.5 & 820 & $\begin{array}{l}\operatorname{cpx}(1) \\
\operatorname{opx}(10) \\
\operatorname{gt}(6) \\
\operatorname{sp}(5) \\
\operatorname{phE}(17)\end{array}$ & $\begin{array}{l}54.0 \\
57.9 \\
39.9 \\
1.68 \\
27.8\end{array}$ & $\begin{array}{l}(20) \\
(12) \\
(54) \\
(24)\end{array}$ & $\begin{array}{l}0.00 \\
0.02 \\
0.34 \\
3.19 \\
0.21\end{array}$ & $\begin{array}{l}(1) \\
(9) \\
(17) \\
(4)\end{array}$ & $\begin{array}{l}0.22 \\
0.26 \\
18.4 \\
1.27 \\
0.09\end{array}$ & $\begin{array}{l}(20) \\
(5) \\
(51) \\
(4)\end{array}$ & $\begin{array}{l}0.12 \\
0.30 \\
2.69 \\
70.1 \\
-\end{array}$ & $\begin{array}{l}(6) \\
(54) \\
(20)\end{array}$ & $\begin{array}{l}12.3 \\
10.5 \\
19.3 \\
9.74 \\
24.1\end{array}$ & $\begin{array}{l}(3) \\
(6) \\
(84) \\
(6)\end{array}$ & $\begin{array}{l}0.46 \\
0.18 \\
1.21 \\
0.18 \\
0.31\end{array}$ & $\begin{array}{l}(1) \\
(13) \\
(2) \\
(5)\end{array}$ & $\begin{array}{l}19.2 \\
31.1 \\
8.39 \\
2.39 \\
35.5\end{array}$ & $\begin{array}{l}(18) \\
(7) \\
(57) \\
(8)\end{array}$ & $\begin{array}{l}14.0 \\
0.16 \\
10.1 \\
- \\
0.06\end{array}$ & $\begin{array}{l}(13) \\
(12)\end{array}$ & $\begin{array}{l}0.38 \\
0.03 \\
0.03 \\
0.14 \\
-\end{array}$ & $\begin{array}{l}(3) \\
(3) \\
(10)\end{array}$ & $\begin{array}{l}0.00 \\
0.01 \\
- \\
- \\
-\end{array}$ & (1) & $\begin{array}{l}0.74 \\
0.84 \\
0.44\end{array}$ & $\begin{array}{l}100.7 \\
100.5 \\
100.4 \\
88.9 \\
88.2\end{array}$ \\
\hline A36 & 7 & 770 & $\begin{array}{l}\text { ol(12) } \\
\text { opx(13) } \\
\text { cpx(3) } \\
\text { gt(5) } \\
\text { phE(20) }\end{array}$ & $\begin{array}{l}37.4 \\
56.7 \\
54.1 \\
39.9 \\
27.0\end{array}$ & $\begin{array}{l}(13) \\
(17) \\
(2) \\
(15) \\
(6)\end{array}$ & $\begin{array}{l}0.09 \\
0.01 \\
0.04 \\
0.39 \\
0.21\end{array}$ & $\begin{array}{l}(5) \\
(2) \\
(5) \\
(10) \\
(2)\end{array}$ & $\begin{array}{l}0.09 \\
0.14 \\
0.38 \\
17.4 \\
0.06\end{array}$ & $\begin{array}{l}(3) \\
(13) \\
(6) \\
(5) \\
(2)\end{array}$ & $\begin{array}{l}- \\
0.16 \\
0.21 \\
1.23 \\
-\end{array}$ & $\begin{array}{l}(4) \\
(1) \\
(17)\end{array}$ & $\begin{array}{l}22.5 \\
11.1 \\
9.55 \\
20.4 \\
29.3\end{array}$ & $\begin{array}{l}(9) \\
(2) \\
(15) \\
(6) \\
(9)\end{array}$ & $\begin{array}{l}0.29 \\
0.16 \\
0.32 \\
0.57 \\
0.42\end{array}$ & $\begin{array}{l}(4) \\
(3) \\
(0) \\
(3) \\
(4)\end{array}$ & $\begin{array}{l}39.2 \\
30.3 \\
16.1 \\
10.7 \\
31.3\end{array}$ & $\begin{array}{l}(18) \\
(19) \\
(16) \\
(7) \\
(9)\end{array}$ & $\begin{array}{l}0.09 \\
0.16 \\
19.1 \\
8.24 \\
0.01\end{array}$ & $\begin{array}{l}(10) \\
(12) \\
(13) \\
(9) \\
(1)\end{array}$ & $\begin{array}{l}- \\
0.02 \\
0.26 \\
0.02 \\
-\end{array}$ & $\begin{array}{l}(2) \\
(7) \\
(2)\end{array}$ & $\begin{array}{l}- \\
0.01 \\
0.01 \\
- \\
-\end{array}$ & $\begin{array}{l}(1) \\
(0)\end{array}$ & $\begin{array}{l}0.75 \\
0.83 \\
0.75 \\
0.48 \\
0.66\end{array}$ & $\begin{array}{l}99.7 \\
98.8 \\
100.0 \\
98.9 \\
88.4\end{array}$ \\
\hline A38 & 7 & 850 & $\begin{array}{l}\operatorname{opx}(12) \\
\operatorname{cpx}(1) \\
\operatorname{gt}(7) \\
\operatorname{phE}(20)\end{array}$ & $\begin{array}{l}56.0 \\
61.2 \\
40.0 \\
28.6\end{array}$ & $\begin{array}{l}(9) \\
(14)\end{array}$ & $\begin{array}{l}0.01 \\
0.01 \\
0.32 \\
0.21\end{array}$ & $\begin{array}{l}\text { (9) } \\
\text { (7) }\end{array}$ & $\begin{array}{l}0.36 \\
0.17 \\
18.6 \\
0.09\end{array}$ & $\begin{array}{l}(11) \\
(6)\end{array}$ & $\begin{array}{l}0.16 \\
0.11 \\
1.75 \\
-\end{array}$ & (54) & $\begin{array}{l}11.5 \\
10.1 \\
20.9 \\
23.4\end{array}$ & $\begin{array}{l}(7) \\
(4)\end{array}$ & $\begin{array}{l}0.20 \\
0.20 \\
1.14 \\
0.29\end{array}$ & $\begin{array}{l}(9) \\
(5)\end{array}$ & $\begin{array}{l}32.1 \\
23.7 \\
9.95 \\
36.4\end{array}$ & $\begin{array}{l}(125) \\
(19)\end{array}$ & $\begin{array}{l}0.15 \\
5.11 \\
7.95 \\
0.07\end{array}$ & $\begin{array}{l}(70) \\
(11)\end{array}$ & $\begin{array}{l}0.05 \\
0.11 \\
0.01 \\
-\end{array}$ & (2) & $\begin{array}{l}0.01 \\
0.01 \\
- \\
-\end{array}$ & (1) & $\begin{array}{l}0.83 \\
0.81 \\
0.46 \\
0.74\end{array}$ & $\begin{array}{l}100.5 \\
100.7 \\
100.6 \\
89.1\end{array}$ \\
\hline
\end{tabular}

ol olivine; opx orthopyroxene; cpx clinopyroxene; gt garnet; sp spinel; cHum clinohumite; TicHum titanoclinohumite; chl chlorite; phE phase E; gl glass.

${ }^{\text {a }}$ Number of microprobe analyses.

${ }^{\mathrm{b}}$ One standard deviation of replicate analyses in terms of least unit cited. 

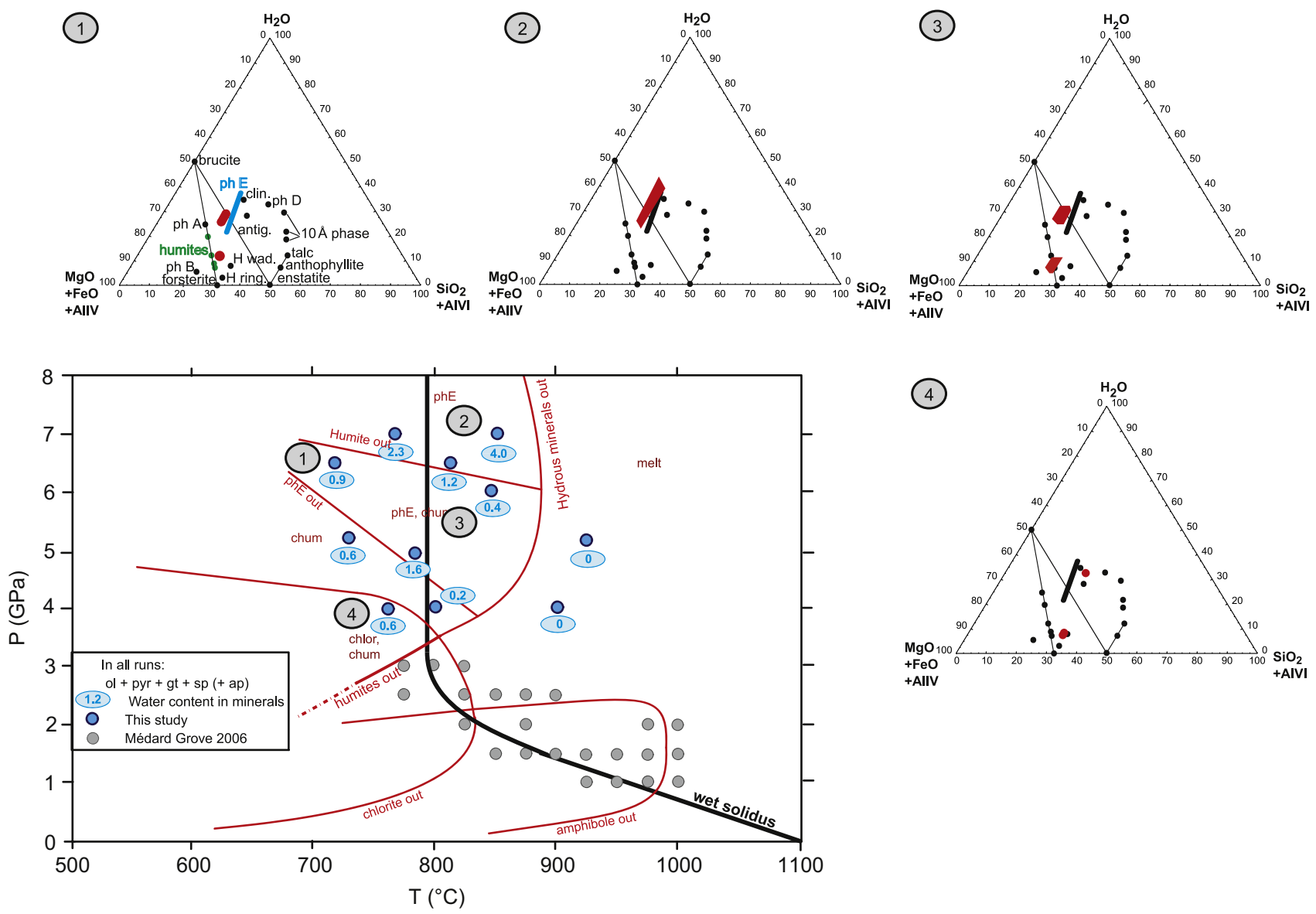

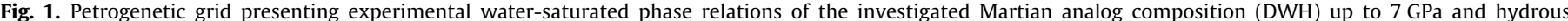

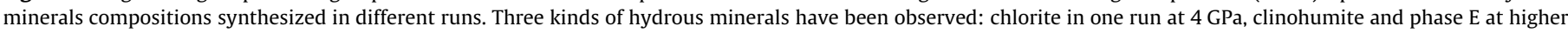

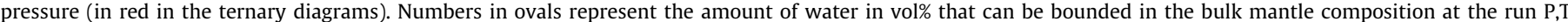

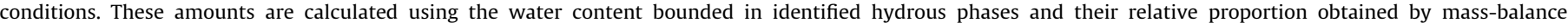
calculations. Stability fields for pressure < 4 GPa come from Médard and Grove (2006).

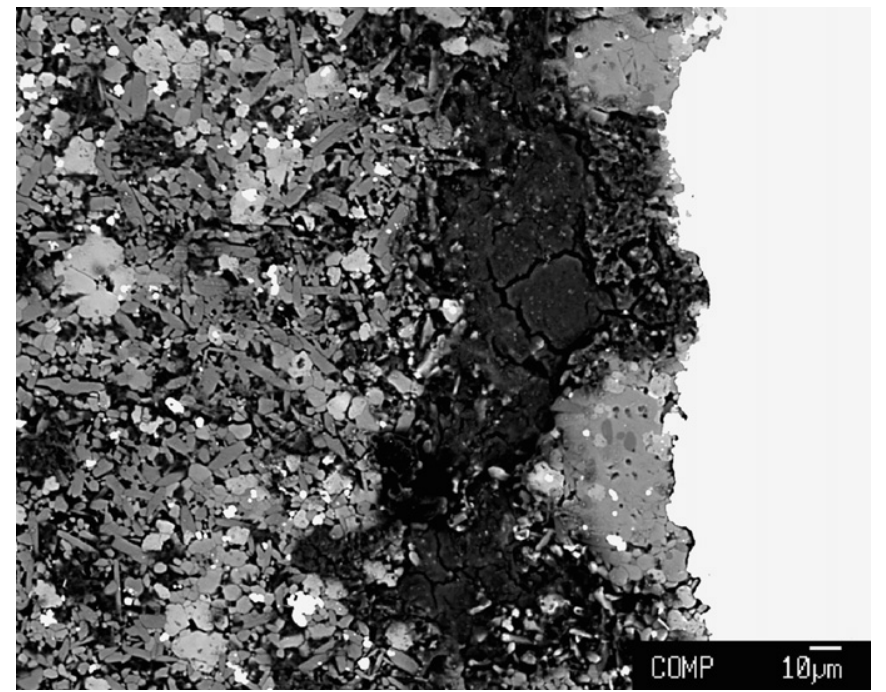

Fig. 2. Back-scattered image of run $\mathrm{A} 25\left(920^{\circ} \mathrm{C}, 5.2 \mathrm{GPa}, 2.5\right.$ day). The quenched melt located close to the capsule wall presents numerous cracks and small bubbleshape cavities, indicating on important amount of dissolved water.

investigated pressure range (Kawamoto and Holloway, 1997; Fumagalli and Poli, 2005). Compositional differences between Earth's and Mars mantles may explain this difference in DHMS stabilities. Actually, the influence of major elements on DHMS stability field has been underscored in a few studies (e.g. Luth, 1995) but the influence of compositional variability remains poorly known (Williams and Hemley, 2001).

\section{Implications for the early Mars evolution}

\subsection{Deep water storage and melting path: comparison with previous models}

Previous investigations of the stability fields of mineral phases in hydrous peridotite can be classified into two groups, depending on the phase stabilities found at high pressure (Fig. 3). Médard and Grove (2006) used results from Fumagalli and Poli (2005) and Schmidt and Poli (1998) to infer hydrous mineral phase relations at $P>3 \mathrm{GPa}$ (Fig. 3B). These two studies found chlorite, phase $\mathrm{A}$ and the $10 \AA$ phase as stable hydrous minerals. The breakdown of chlorite and the $10 \AA$ phase are in agreement with the recent study by Dvir et al. (2011). However, our results are more consistent with the studies by Kawamoto and Holloway (1997) and Kawamoto et al. (1996). These two studies did not synthesize $10 \AA$ phase or phase $A$ but observed the presence of humite minerals and phase $\mathrm{E}$ (Fig. 3A and C). The differences in hydrous mineral stabilities are most likely explained by the difference in bulk chemical composition of the starting peridotite. The starting compositions of Kawamoto and coworkers has an Mg\# of 78-81, 
A

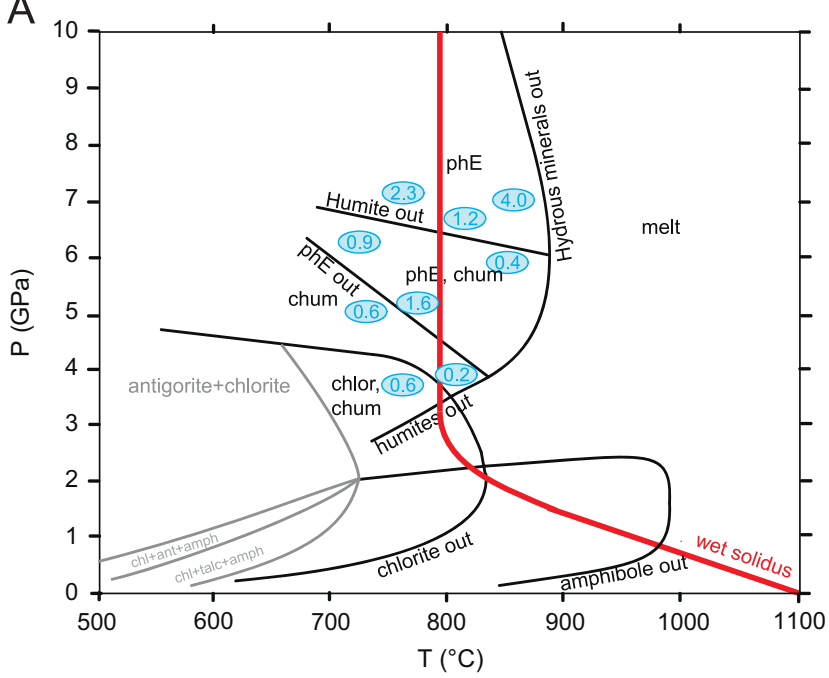

B
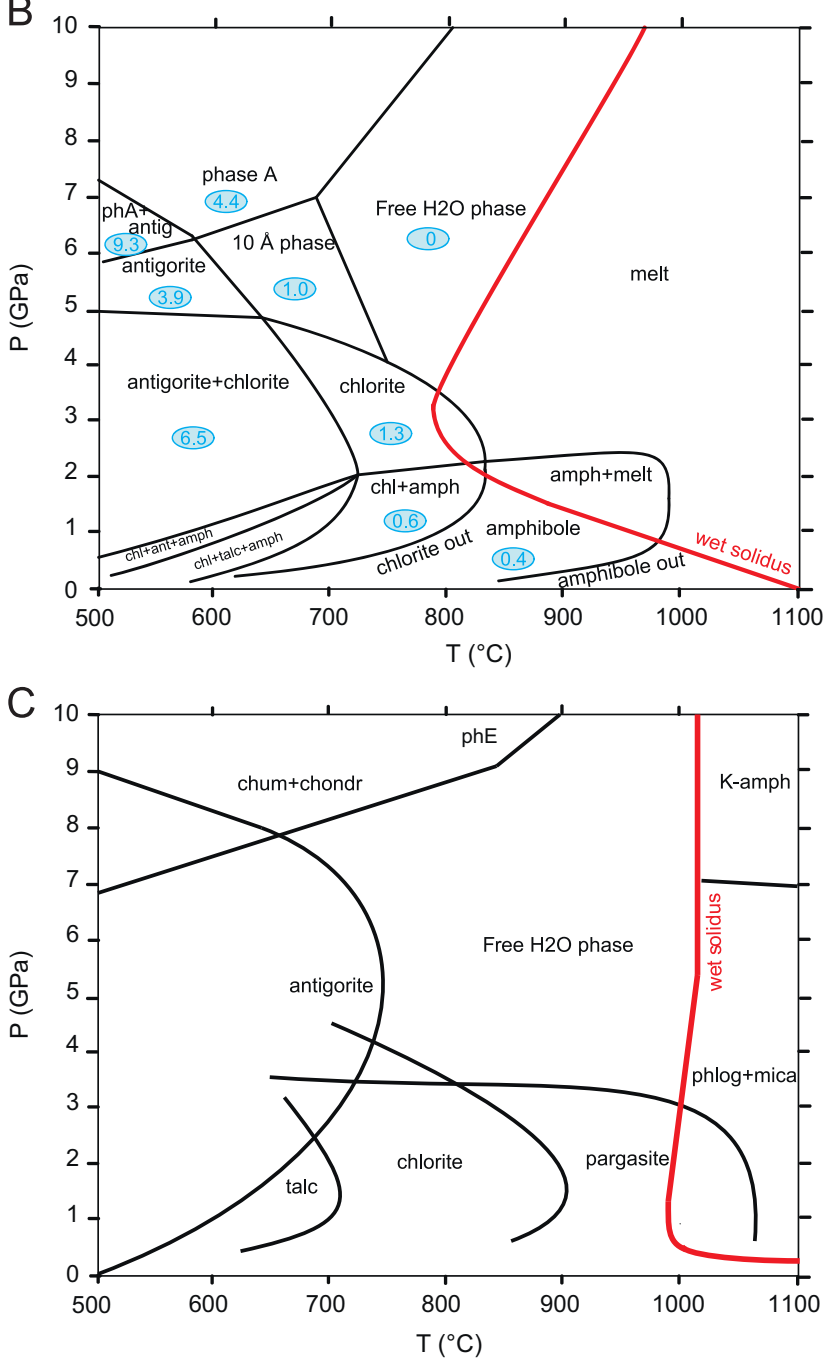

Fig. 3. Stability diagrams for hydrous phases as a function of pressure and temperature for water-saturated peridotite mineralogy. (A) Fe-rich peridotite (DWH), this study. Chlorite (chlor), clinohumite (chum) and phase E (phE) have been identified at pressures up to $7 \mathrm{GPa}$. (B) Same composition, Médard and Grove (2006). Experiments constraints stability fields up to $3 \mathrm{GPa}$. Hydrous phase at higher pressure are predicted based on previous studies on (Mg-rich) Earth peridotite (see text for details). (C) Fe-rich peridotite (Kilborne Hole), Kawamoto and Holloway, 1997. This composition is closer to the one investigated in the present study and led to the synthesis of similar hydrous phases (humites and phase E). which is closer to our composition ( $\mathrm{Mg} \#=75)$. The compositions used by Fumagalli and Poli (2005) has Mg\#=83-91. Therefore, it seems that the humite minerals and phase $\mathrm{E}$ are favored in ironrich peridotites compared to the one of phase $A$ and $10 \AA$ phase, stable in compositions with higher Mg\#.

Médard and Grove (2006) estimated the position of the wet solidus by extrapolating the Mysen and Boettcher (1975) watersaturated solidus. Our results determine the wet solidus position directly and show that there is no pressure effect between 3 and $7 \mathrm{GPa}$, a result similar to that found by Kawamoto and Holloway (1997). The differences in the water-saturated solidus as well as in mineral phase stabilities have significant implications regarding the amount of water that can be stored at high pressure and the melting processes within Mars. First, the absence of pressure effect on the position of the wet solidus implies that melting at pressures $>3 \mathrm{GPa}$ occurs at temperatures distinctly cooler than what was inferred by Médard and Grove (2006). For example, at $7 \mathrm{GPa}$, our experiments show that melting starts at $\sim 780^{\circ} \mathrm{C}$, which is $\sim 100{ }^{\circ} \mathrm{C}$ cooler than previous predictions (Fig. $3 \mathrm{C}$ and $\mathrm{B}$ ). In terms of accretion processes, this means that the formation of a low-degree $\mathrm{H}_{2} \mathrm{O}$-rich melt, and thus the initiation of a hydrous component to the developing magma ocean, could be initiated at a temperature lower than previously predicted. As discussed below, these new constraints can be combined with thermal models to predict the evolution of Martian magma ocean. Secondly, the absence of phase $A$ and $10 \AA$ phase and the presence of humite minerals together with phase $\mathrm{E}$ demonstrate a higher storage capacity of water in the Martian mantle than predicted by Médard and Grove (2006) (Fig. 3A and B). At temperatures close to the water-saturated solidus, our data show that up to $4 \mathrm{wt} \%$ water can be stored in the mantle, because of the abundance of phase E. In contrast, Médard and Grove (2006) predicted no hydrous minerals and only the presence water as a supercritical fluid phase. These discrepancies will modify the amount of water that can be stored in the Martian mantle and mobilized during melting. In the next section we will explore an early wet accretion model and the subsequent melting differentiation of Mars.

\subsection{Constraints on early hydrous melting from time scales of Mars accretion}

Several important criteria need to be met if early hydrous melting processes are to be of importance in the Martian mantle: (1) the accreting bodies must be cold so that the water they contain has not been degassed at the time of their accretion and (2) the thermal evolution of proto-Mars needs to allow water brought in by the accreting materials to be stored inside the planet before melting begins so that water can participate in the differentiation process. Hydrous melting can occur in two ways. Vapor-saturated melting in the presence of an $\mathrm{H}_{2} \mathrm{O}$-rich supercritical fluid can occur if hydrous minerals trapped in the deep interior of the planet break down to anhydrous silicates and release their structurally bound water. Hydrous melting in the absence of excess water can also occur when hydrous minerals stable above the water-saturated solidus breakdown and release water. There are three regions in the phase diagram (Fig. 3A) where this type of melting can occur at the termination of amphibole, chlorite and humite+phase E stability. Constraints on the water content come from the model of the bulk composition of Mars by Lodders and Fegley (1997) that uses oxygen isotopes to estimate the types and proportions of chondritic material that make up Mars. The thermal evolution of Mars can be inferred from the models of accretion (Senshu et al., 2002) and from constraints on the timing of core formation on Mars that is assumed to occur after the planet has accreted. 
a

b
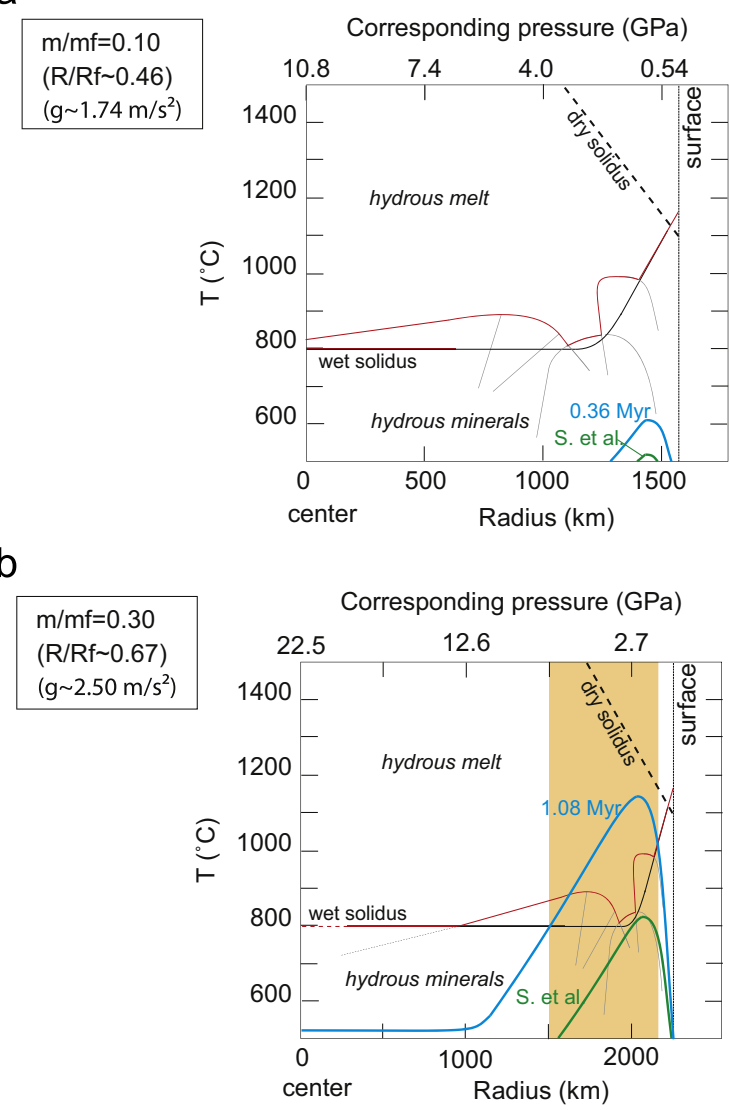

C

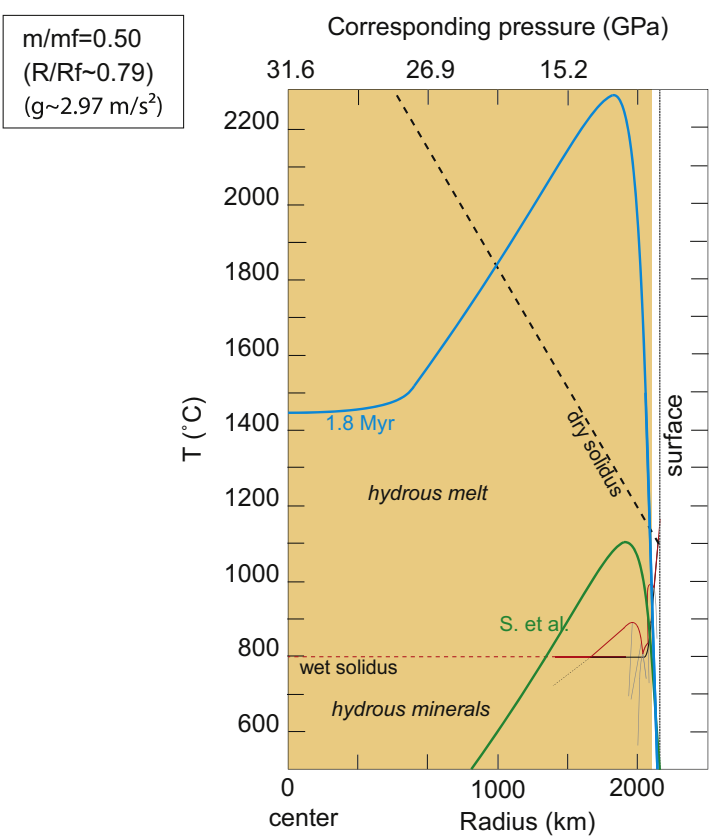

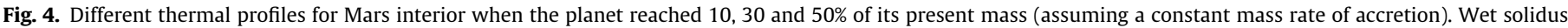

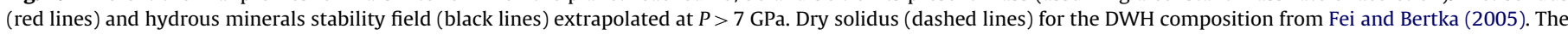

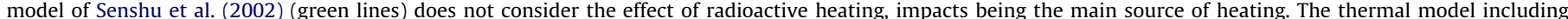

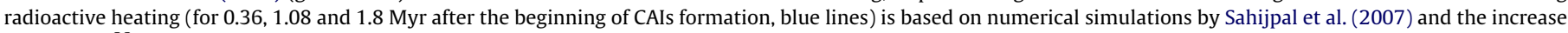

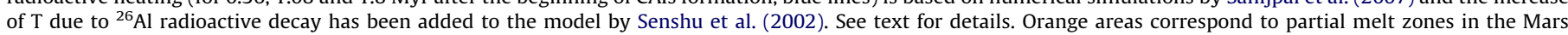

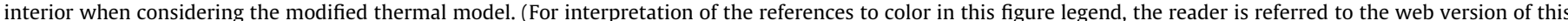
article.)

Evidence from the Hf/W isotopic system indicates that Mars accreted rapidly. Kleine et al. (2004), Jacobsen (2005), Foley et al. (2005) and Nimmo and Kleine (2007) estimate core formation ages between 0 and $15 \mathrm{Myr}$ after the formation of CAIs in the early solar nebula. The ranges in these estimates arise from the range in Hf abundance in different chondrites (Nimmo and Kleine, 2007). Dauphas and Pourmand (2011) recognize this uncertainty and refined the $\mathrm{Hf} / \mathrm{W}$ abundances in Mars mantle allowing a tighter constraint on core formation and infer that Mars accreted to half its present size in $1.8 \mathrm{Myr}$ or less. With such a short time between the formation of the elements in the solar nebula and assembly of Mars, ${ }^{26} \mathrm{Al}$ decay to ${ }^{26} \mathrm{Mg}$ becomes an important heat source in the accretion of Mars (e.g. Gosh and McSween, 1998) and therefore, the accreting proto-planet will contain sufficient ${ }^{26} \mathrm{Al}$ to become a significant heat source.

\subsection{Models of Mars accretion}

Two thermal models are presented in Fig. 4 at different time steps in the accretion process. These steps concern the early accretion of Mars (i.e. before and just after melting occurred), when $\mathrm{H}_{2} \mathrm{O}$ was potentially kept in the interior of the planet. This approach considers that Mars accreted mostly from undifferentiated materials, which may be possible, particularly under cold conditions. But it does not apply in case sufficient time has elapsed so that the small accreting bodies have heated up and de-hydrated.
One model is that of Senshu et al. (2002) who consider the heating that occurs from impact heating during rapid accretion (1 Myr) and neglects radioactive heating. The second model includes the effect of heating from decay of ${ }^{26} \mathrm{Al}$ and ${ }^{60} \mathrm{Fe}$ in addition to heating from accretion (adapted after Sahijpal et al., 2007). The thermal model that includes accretion and radioactive heating was estimated by adding the heating expected from ${ }^{26} \mathrm{Al}$ decay (Sahijpal et al., 2007) to the Senshu et al. (2002) thermal model. The addition of temperature from radioactivity to the Senshu et al.'s model represents a simple approach to the modeling of Mars early evolution. However, it provides a first estimate of the effect of both radioactive and impact heating on the thermal history of Mars. We have adjusted the accretion time scale to account for core formation at $1.8 \mathrm{Myr}$ as inferred from Hf/W isotopic constraints when Mars reached about half of its present mass (Dauphas and Pourmand, 2011). The temperature distribution in the planet is superimposed on our hydrous Martian phase equilibria (Fig. 4a) at a time when $10 \%$ of the mass of Mars has accreted $(m / m f=0.1)$ and it has reached a radius that is $46 \%$ of its final radius $(R / R f=0.46)$. At this stage in the accretion process the deep interior of the proto-Mars will be cool in both thermal models and all hydrous minerals added during accretion will be stable or metastable throughout the planet. An estimate of the bulk water content of the accreting material can be obtained using the Lodders and Fegley (1997) oxygen-isotope model for Mars bulk composition and the water content of the chondrites (Jarosewich, 1990). The chondrite mix inferred by Lodders and 
Fegley (1997) is $85 \% \mathrm{H}, 11 \% \mathrm{CV}$ and $4 \% \mathrm{CI}$. We assume the $\mathrm{H}$ chondrites have lost some of the water that is found in the lowgrade $\mathrm{H} 3$ chondrites and infer an $\mathrm{H}_{2} \mathrm{O}$ content of $0.2 \mathrm{wt} \%$. CV and $\mathrm{CI}$ carbonaceous chondrites contain 2.3 and $16.9 \mathrm{wt} \% \mathrm{H}_{2} \mathrm{O}$, respectively and carry the bulk of the water in the accreting material. The bulk water content of the chondrite mix is $1.1 \mathrm{wt} \% \mathrm{H}_{2} \mathrm{O}$ that is buried in the Martian interior.

The next stage in the early accretion of Mars occurs at a time when the proto-Mars has reached $30 \%$ of its final mass and $R / R f=0.67$ (Fig. 4b). In the model that considers both accretion and radioactive heat sources Mars is above the water-saturated solidus from 100 to $720 \mathrm{~km}$ depth and the temperature drops in the deep interior to just above $500{ }^{\circ} \mathrm{C}$. Within the molten zone melt fraction will increase with decreasing depth, but melt fractions will probably remain low ( $<20 \%$ for Earth-like peridotite, Gaetani and Grove, 2003 but this estimate is probably a bit higher for more fertile (Fe-rich) peridotite) and the water content of the melt will be high. The mixture of chondritic material in the deep interior beneath the melt zone will be at high enough temperature so that it will react and recrystallize to the stable mineral assemblage (anhydrous silicates + humite minerals + phase E) and the storage capacity for $\mathrm{H}_{2} \mathrm{O}$ in the Martian mantle is sufficient to keep the water bound stably in crystalline solids. In the Senshu et al. (2002) thermal model the heat derived from accretion has heated the interior so that it has just reached the water-saturated solidus at a depth of $200 \mathrm{~km}$. This is the last stage at which water can be trapped in significant amounts during the accretion process, because the temperature at shallow, near-surface depths increases rapidly and water-bearing minerals become unstable. At this point, the amount of water stored in Mars could be as much as $7.2 \times 10^{21} \mathrm{~kg}$ or 5.1 Earth oceans of water using the chondrite mix of Lodders and Fegley (1997).

The critical stage in the early accretion of Mars occurs at a time when the proto-Mars has reached $50 \%$ of its final mass and $R / R f=0.79$ (Fig. 4c). In the model that considers both accretion and radioactive heat sources the interior of Mars is now above the dry solidus from 50 to $1400 \mathrm{~km}$ depth. A low- $\mathrm{H}_{2} \mathrm{O}$ melt will be present and melting extent will be high. This is the time of core formation based on Dauphas and Pourmand (2011) Hf/W isotopic constraint and core formation would be facilitated in a wet magma ocean at this time. The temperature of the outer part of Mars has increased dramatically from the effect of ${ }^{26} \mathrm{Al}$ decay and accretionary heating. If only the accretionary heating is considered, a shallower outer zone of Mars is above the watersaturated solidus from 100 to $720 \mathrm{~km}$ depth and the temperature drops in the deep interior and remains low, much like the situation in Fig. $4 \mathrm{~b}$ where ${ }^{26} \mathrm{Al}$ is contributing heat to raise the temperature of the interior. Senshu et al. (2002) show that this temperature profile rapidly develops into one where a magma ocean envelops the planet. From this time of Mars accretion, it is worth noticing that gravitational potential energy released by metal segregation into the core (Ricard et al., 2009) probably becomes an important energetic contribution. However, we assume that it has little influence in the early Mars since it is likely that core formation really occurred only when hydrous melting was initiated.

Based on our experiments and existing thermal models, Fig. 4 clearly underlines the critical influence of ${ }^{26} \mathrm{Al}$ radioactive heating as part of the thermal evolution of the planet. These results stress the importance of the contributions from radioactive decay in simulations of the thermal evolution of planetesimals that accrete rapidly and early on in solar system evolution. The model that includes radioactive heating (Fig. 4) predicts that a significant part of the outer portions of proto-Mars will be above the water-saturated solidus, just before the planet reached half of its present mass. This is significantly earlier than what is predicted by Senshu et al.
(2002) who propose that the magma ocean forms of when the planet reached $\sim 95 \%$ of its current mass. It is worth noting that, Senshu et al. (2002) model is a scenario of dry accretion (solidus determined by Fei and Bertka, 2005). An interesting consequence of hydrous melting is that a significant amount of melt has formed in the presence of water by the time Mars is 50\% accreted and this is when the Hf/W isotopic system predicts the onset of core formation.

Fig. 4 shows that thermal models agree on an initiation of melting at shallow depth (between 1 and $3 \mathrm{GPa}$ ), regardless of the source of heating (impacts, or impacts $+{ }^{26} \mathrm{Al}$ ). But radioactive heating combined with the vertical slope of the wet solidus in pressuretemperature space to at least $7 \mathrm{GPa}$ (Fig. 3) implies that as soon as melting starts, it affects most of the interior of proto-Mars and is not limited to a shallow depth range. This conclusion is a major contrast between our results and the study by Médard and Grove (2006) of cold interior lasting for a large part of Mars accretion.

\subsection{Implications for magma ocean and fate of water}

The formation of a magma ocean in an initially hydrous embryo raises the question of the fate of water during accretion and differentiation. Water was driven out of hydrous minerals to a melt phase when the increase in temperature crosses the wet solidus, i.e. at $m / m f \sim 0.50$. This occurs as the core forms. Our current knowledge of Mars history cannot exclude that part of the initial water budget might have been partitioned into metallic blobs and segregated into the core. Mars differentiation had likely already started at the time of magma ocean formation (e.g. Righter and Chabot, 2011). Actually, the formation of a buoyant solid silicate layer, suggested as a possible differentiation mechanism to trap metal into the forming core, could hamper the mobility of hydrogen towards the surface and thus limit its degassing. Experimental studies of metal/silicate partitioning of hydrogen and other light elements have observed that a significant amount of these elements can be incorporated into molten iron (Okuchi, 1997; Terasaki et al., 2011). The process of hydrogen and other light elements segregation into metallic core has been proposed to explain the Earth's differentiation and density deficit of its core (Poirier, 1994; Okuchi, 1997) and may be relevant for Mars as well.

As the Mars magma ocean evolves, the combined effects of accretionary and radioactive heating will lead to the formation of a planet-wide magma ocean. This ocean will cool (Elkins-Tanton et al., 2003) and crystallize from the bottom up. Water will be concentrated in the residual liquid and lost from the surface of the ocean as convection transports the dissolved water to the surface where vapor-saturation and degassing processes lead to a significant loss of water from the forming planet, into a protoatmosphere and/or directly released to the atmosphere (ElkinsTanton, 2011). If our assumptions about the amount of water that can be trapped during accretion are correct, the amount of water that is degassed into the atmosphere is enormous (5.1 Earth oceans). Even if we assume that the $\mathrm{CV}$ and $\mathrm{CI}$ carbonaceous chondrites had degassed prior to incorporation into Mars and contained only a small amount of water (say $0.2 \mathrm{wt} \%$, like the assumption we made for the dominant $\mathrm{H}$ chondrite component) Mars would still end up trapping $5 \mathrm{wt} \%$ of an Earth ocean $\left(3.9 \times 10^{20} \mathrm{~kg}\right)$. Elkins-Tanton (2008) has discussed the importance of small amounts of accretion-trapped water during solidification of planet-wide magma ocean, and a better knowledge of the amount of water trapped in Mars provides important constraints of the cooling and solidification. We suggest here that water also plays an important role in the early evolution of planet-wide magma oceans and may have promoted early core 
formation on Mars and accelerated and extended melting over a large portion of Mars interior.

\section{Concluding remarks}

This study presents experimental data that provide new insights into the role of water's influence on planetary melting and differentiation processes during Mars accretion. We suggest that water can play an important role in promoting early melting of the Martian mantle and may also serve to accelerate the early core formation process that is a unique and important chemical differentiation event.

\section{Acknowledgments}

This work is supported by the NASA Mars fundamental research program (grant \#NNX07AR02G). We are grateful to Tony Withers, David Kohlstedt and Mike Krawczynski for their help on multi-anvil experiments. Tony Withers, Brent Grocholski and Dan Shim are thanked for Raman analyses. Comments from two anonymous reviewers helped to improve the manuscript.

\section{Appendix A. Supplementary material}

Supplementary data associated with this article can be found in the online version at http://dx.doi.org/10.1016/j.epsl.2012. 03.030 .

\section{References}

Agee, C.B., Draper, D.S., 2004. Experimental constraints on the origin of Martian meteorites and the composition of the Martian mantle. Earth Planet. Sci. Lett. 224, 415-429.

Armstrong, J.T., 1995. Citzaf-a package of correction programs for the quantitative electron microbeam X-ray analysis of thick polished materials, thin-films, and particles. Microbeam. Anal. 4, 177-200.

Bertka, C.M., Fei, Y., 1997. Mineralogy of the Martian interior up to core-mantle boundary pressures. J. Geophys. Res. 102 (B3), 5251-5264.

Bertka, C.M., Holloway, J.R., 1994a. Anhydrous partial melting of an ironrich mantle. 1. Subsolidus phase assemblages and partial melting phaserelations at 10 to 30 Kbar. Contrib. Mineral Petrol 115 (3), 313-322.

Bertka, C.M., Holloway, J.R., 1994b. Anhydrous partial melting of an ironrich mantle. 2. Primary melt compositions at 15 Kbar. Contrib. Mineral. Petrol. 115 (3), 323-338.

Bohlen, S.R., Boettcher, A.L., 1982. The quartz-reversible-coesite transformation-a precise determination and the effects of other components. J. Geophy. Res. 87 (NB8), 7073-7078.

Boyd, F.R., England, J.L., 1960. Apparatus for phase equilibrium measurements of pressures up t o 50 kbars and temperatures up to $1750^{\circ} \mathrm{C}$. J. Geophys. Res. 65, 741-748.

Carr, M.H., Head, J.W., 2010. Geologic history of Mars. Earth Planet. Sci. Lett. 294 (3-4), 185-203.

Comodi, P., Cera, F., Nazzareni, S., Dubrovinsky, L., 2007. Raman spectroscopy of the 10-A phase at simultaneously HP-HT. Eur. J. Miner. 19, 623-629.

Dauphas, N., Pourmand, A., 2011. Hf-W-Th evidence for rapid growth of Mars and its status as a planetary embryo. Nature $473,489-493$.

Drake, M.J., 2005. Origin of water in the terrestrial planets. Meteor. Planet. Sci. 40 (4), 519-527.

Dreibus, G., Wänke, H., 1985. Mars, a volatilerich planet. Meteoritics 20 (2), 367-381.

Dvir, O., Pettke, T., Fumagalli, P., Kessel, R., 2011. Fluids in the peridotite-water system up to $6 \mathrm{GPa}$ and $800{ }^{\circ} \mathrm{C}$ : new experimental constraints on dehydration reactions. Contrib. Mineral. Petrol. 161, 829-844.

Elkins-Tanton, L.T., 2008. Linked magma ocean solidification and atmospheric growth for Earth and Mars. Earth Planet. Sci. Lett. 271, 181-191.

Elkins-Tanton, L.T., 2011. Formation of early water oceans on rocky planets. Astophys. Space Sci. 332, 359-364.

Elkins-Tanton, L.T., Parmentier, E.M., Hess, P.C., 2003. Magma ocean fractional crystallization and cumulate overturn in terrestrial planets: implications for Mars. Meteor. Planet. Sci. 38 (12), 1753-1771.

Fei, Y., Bertka, C., 2005. The interior of Mars. Science 308, 1120-1121.

Filiberto, J., Treiman, A.H., Le, L., 2008. Crystallization experiments on a Gusev Adirondack basalt composition. Meteorit. Planet. Sci. 43 (7), 1137-1146.
Filiberto, J., Dasgupta, R., Kiefer, W.S., Treiman, A.H., 2010. High pressure, nearliquidus phase equilibria of the Home Plate basalt Fastball and melting in the Martian mantle. Geophys. Res. Lett. 37, L13201.

Filiberto, J., Treiman, A.H., 2009. Martian magmas contained abundant chlorine but little water. Geology 37 (12), 1087-1090.

Foley, C.N., Wadhwa, M., Borg, L.E., Janney, P.E., Hines, R., Grove, T.L., 2005 The early differentiation history of Mars from $182 \mathrm{~W}-142 \mathrm{Nd}$ isotope systematics in the SNC meteorites. Geochim. Cosmochim. Acta 69 (18) 4557-4571.

Frost, R.L., Palmer, S.J., Bouzaid, J.M., Reddy, B.J., 2007. A Raman spectroscopic study of humite minerals. J. Raman Spectrosc. 38, 68-77.

Fumagalli, P., Poli, S., 2005. Experimentally determined phase relations in hydrous peridotites to $6.5 \mathrm{GPa}$ and their consequences on the dynamics of subduction zones. J. Petrol 46 (3), 555-578.

Gaetani, G., Grove, T.L., 2003. Experimental constraints on melt generation in the mantle wedge. In Inside the Subduction Factory. In: Eiler, J. (Ed.), American Geophysical Monograph, 138; 2003, pp. 107-133.

Gosh, A., McSween Jr, H.Y., 1998. A thermal model for the differentiation of planetesimal 4 Vesta, based on radiogenic heating. Icarus 134, 187-206.

Hart, S.R., Zindler, A., 1986. In search of a bulk-Earth composition. Chem. Geol. 57, 247-267.

Hernlund, J., Leinenweber, K., Locke, D., Tyburczy, J.A., 2006. A numerical model for steady-state temperature distributions in solid-medium high-pressure cell assemblies. Am. Mineral. 91 (2-3), 295-305.

Horiuchi, H., Morimoto, N., Yamamoto, K., Akimoto, S., 1979. Crystal structure of $2 \mathrm{Mg}_{2} \mathrm{SiO}_{4} \cdot 3 \mathrm{Mg}(\mathrm{OH})_{2}$, a new high-pressure structure type. Am. Mineral. 64, 593-598.

Jacobsen, S.B., 2005. The Hf-W isotopic system and the origin of the Earth and Moon. Annu. Rev. Earth Planet. Sci 33, 531-570.

Jarosewich, E., 1990. Chemical analyses of meteorites: a compilation of stony and iron meteorite analyses. Meteoritics 25, 323-337.

Kanzaki, M., 1989. High pressure phase relations in the system $\mathrm{MgO}-\mathrm{SiO}_{2}-\mathrm{H}_{2} \mathrm{O}$. Eos 70 (508).

Kanzaki, M., 1991. Stability of hydrous magnesium silicates in the mantle transition zone. Ph. Earth Planet. Int. 66, 307-312.

Kawamoto, T., Hervig, R.L., Holloway, J.R., 1996. Experimental evidence for a hydrous transition zone in Earth's early mantle. Earth Planet. Sci. Lett. 142, 587-592.

Kawamoto, T., Holloway, J.R., 1997. Melting temperature and partial melt chemistry of $\mathrm{H}_{2} \mathrm{O}$-saturated mantle peridotite to 11 gigapascals. Science 276, 240-243.

Kleine, T., Mezger, K., Munker, C., Palmer, H., Bischoff, A., $2004 .{ }^{182} \mathrm{Hf}-{ }^{182} \mathrm{~W}$ isotope systematics of chondrites, eucrites and martian meteorites: chronology of core formation and early mantle differentiation in Vesta and Mars. Geochem. Cosmochim. Acta 68, 2935-2946.

Kudoh, Y., Finger, L.W., Hazen, R.M., Prewitt, C.T., Kanzaki, M., Veblen, D.R., 1993. Phase E: a high pressure hydrous silicate with unique crystal chemistry. Phys. Chem. Miner. 19, 357-360.

Kushiro, I., Syono, Y., Akimoto, S.-i, 1968. Melting of a peridotite nodule at high pressures and high water pressures. J. Geophy. Res. 73 (18), 6023-6029.

Lindsley, D.H., Dixon, S.A., 1976. Diopside-enstatite equilibria at 850degrees to $1400^{\circ} \mathrm{C}, 5$ to $35 \mathrm{~kb}$. Am. J. Sci $276(10), 1285-1301$.

Lodders, K., Fegley Jr., B., 1997. An oxygen isotope model for the composition of Mars. Icarus 126, 373-394.

Lunine, J.I., Chambers, J., Morbidelli, A., Leshin, L.A., 2003. The origin of water on Mars. Icarus 165, 1-8.

Luth, R.W., 1995. Is phase A relevant to the Earth's mantle? Geochim. Cosmochim. Acta 59, 679-682.

Marty, B., Marti, K., 2002. Signatures of early differentiation of Mars. Earth Planet. Sci. Lett. 196 (3-4), 251-263.

McSween, H.Y., Grove, T.L., Lentz, R.C.F., Dann, J.C., Holzheid, A.H., Riciputi, L.R. Ryan, J.G., 2001. Geochemical evidence for magmatic water within Mars from pyroxenes in the Shergotty meteorite. Nature 409 (6819), 487-490.

Médard, E., Grove, T.L., 2006. Early hydrous melting and degassing of the Martian interior. J. Geophy. Res. 111, E11003.

Mernagh, T.P., Liu, L.-G., 1998. Raman and infrared spectra of Phase E, a plausible hydrous phase in the mantle. Can. Miner. 36, 1217-1223.

Minitti, M.E., Rutherford, M.J., 2007. Genesis of the Mars Pathfinder "sulfur-free" rock from SNC parental liquids. Geochim. Cosmochim. Acta 64 (14), 2535-2547.

Monders, A.G., Médard, E., Grove, T.L., 2007. Phase equilibrium investigations of the Adirondack class basalts from the Gusev plains, Gusev Crater. Mars. Meteorit. Planet. Sci. 42, 131-148.

Musselwhite, D.S., Dalton, H.A., Kiefer, W.S., Treiman, A.H., 2006. Experimental petrology of the basaltic shergottite Yamato980459: Implications for the thermal structure of the Martian mantle. Meteorit. Planet. Sci. 41, 1271-1290.

Mysen, B.O., Boettcher, A.L., 1975. Melting of a hydrous mantle: I. Phaserelations of natural peridotite at high pressures and temperatures with controlled activities of water, carbon dioxide, and hydrogen. J. Petrol. 16, 520-548.

Mysen, B.O., Virgo, D., Popp, R.K., Bertka, C.M., 1998. The role of $\mathrm{H}_{2} \mathrm{O}$ in Martian magmatic systems. Am. Miner. 83 (9-10), 942-946.

Nimmo, F., Kleine, T., 2007. How rapidly did Mars accrete? Uncertainties in the Hf-W timing of core formation. Icarus 191, 497-504.

Okuchi, T., 1997. Hydrogen partitioning into molten iron at high pressure: implications for Earth's core. Science 278, 1781-1784.

Poirier, J.P., 1994. Light elements in the Earth's outer core: a critical review. Ph. Earth Planet. Int. 85, 319-337. 
Ricard, Y., Srámek, O., Dubuffet, F., 2009. A multi-phase model of runaway core-mantle segregation in planetary embryos. Earth Planet. Sci. Lett. 284, 144-150.

Righter, K., Chabot, N.L., 2011. Moderately and slightly siderophile element constraints on the depth and extent of melting in early Mars. Mekorit. Planet. Sci. 46, 157-176

Sack, R.O., Ghiorso, M.S., 1991. Chromian spinels as petrogenetic indicators; thermodynamics and petrological applications. Am. Miner. 76 (5-6), 827-847.

Sahijpal, S., Soni, P., Gupta, G., 2007. Numerical simulations of the differentiation of accreting planetesimals with ${ }^{26} \mathrm{Al}$ and ${ }^{60} \mathrm{Fe}$ as the heat sources. Meteorit. Planet. Sci. 42 (9), 1529-1548.

Schmidt, M.W., Poli, S., 1998. Experimentally based water budgets for dehydrating slabs and consequences for arc magma generation. Earth Planet. Sci. Lett. 163, 361-379.
Senshu, H., Kuramoto, K., Matsui, T., 2002. Thermal evolution of a growing Mars. J. Geophy. Res. 107 (E12), 5118.

Terasaki, H., Kamada, S., Sakai, T., Ohtani, E., Hirao, N., Ohishi, Y., 2011. Liquidus and solidus temperatures of a Fe-O-S alloy up to the pressures of the outer core: implication for the thermal structure of the Earth's core. Earth Planet. Sci. Lett. 304, 559-564.

Walker, D., 1991. Lubrication, gasketing, and precision in multianvil experiments. Am. Miner. 76 (7-8), 1092-1100.

Williams, Q., Hemley, R.J., 2001. Hydrogen in the deep Earth. Annu. Rev. Earth Planet. Sci. 29, 365-418.

Yagi, T., Akaogi, M., Shimomura, O., Suzuki, T., Akimoto, S., 1987. In situ observation of the olivine-spinel phase transformation in Fe2SiO4 using synchrotron radiation. J. Geophy. Res. 92, 6207-6213. 